\title{
Priorities for research in child maltreatment, intimate partner violence and resilience to violence exposures: results of an international Delphi consensus development process
}

\author{
C Nadine Wathen ${ }^{1 *}$, Jennifer CD MacGregor ${ }^{1}$, Joanne Hammerton ${ }^{1}$, Jeffrey H Coben², Helen Herrman², \\ Donna E Stewart ${ }^{4}$ and Harriet L MacMillan ${ }^{5}$ for the PreVAiL Research Network
}

\begin{abstract}
Background: Intimate partner violence (IPV) and child maltreatment (CM) are major global public health problems. The Preventing Violence Across the Lifespan (PreVAiL) Research Network, an international group of over 60 researchers and national and international knowledge-user partners in CM and IPV, sought to identify evidence-based research priorities in IPV and CM, with a focus on resilience, using a modified Delphi consensus development process.

Methods: Review of existing empirical evidence, PreVAiL documents and team discussion identified a starting list of 20 priorities in the following categories: resilience to violence exposure (RES), CM, and IPV, as well as priorities that cross-cut the content areas (CC), and others specific to research methodologies (RM) in violence research. PreVAiL members $(\mathrm{N}=47)$ completed two online survey rounds, and one round of discussions via three teleconference calls to rate, rank and refine research priorities.
\end{abstract}

Results: Research priorities were: to examine key elements of promising or successful programmes in RES/CM/IPV to build intervention pilot work; CC: to integrate violence questions into national and international surveys, and RM: to investigate methods for collecting and collating datasets to link data and to conduct pooled, meta and sub-group analyses to identify promising interventions for particular groups.

Conclusions: These evidence-based research priorities, developed by an international team of violence, gender and mental health researchers and knowledge-user partners, are of relevance for prevention and resilience-oriented research in the areas of IPV and CM.

\section{Background}

Intimate partner violence (IPV) and child maltreatment $(\mathrm{CM})$ are major global public health problems $[1,2]$. Despite the fact that exposure to violence is recognized as highly correlated with mental health problems [3-7] there has been little opportunity for investigators in mental health and addictions, CM and IPV to collaborate to develop and test approaches to reduce violence and associated impairment. Furthermore, with the

\footnotetext{
* Correspondence: nwathen@uwo.ca

${ }^{1}$ Faculty of Information \& Media Studies, Western University, London, ON, Canada

Full list of author information is available at the end of the article
}

exception of some specific interventions for child maltreatment [8] and even fewer for IPV $[9,10]$, there remains a paucity of research evidence about effective interventions for family violence [11-13], and specifically interventions that focus on resilience in the face of these exposures [14,15].

Our international team of over 60 collaborating investigators and policy partners has been funded by the Canadian Institutes for Health Research's (CIHR) Institute for Gender and Health and Institute of Neurosciences, Mental Health and Addictions to establish the PreVAiL Research Network, a Centre for Research Development in Gender, Mental Health and Violence Across the 
Lifespan. PreVAiL has three main objectives: 1) to increase understanding and knowledge about the links between mental health impairment, gender and exposure to child maltreatment and IPV, both in Canada and internationally; 2) to develop and test interventions to prevent or reduce child maltreatment, IPV and subsequent mental health problems; and 3) to develop and promote an integrated research and knowledge translation and exchange (KTE) agenda among a network of established, new and emerging researchers and key stakeholders. A key feature of PreVAiL is an emphasis on understanding and articulating factors to enhance resilience among those exposed to violence, using a genderbased approach. The team includes a broad range of collaborating academic investigators with expertise in our key content areas, and partnerships with national and supra-national Canadian and international organizations such as the Public Health Agency of Canada, the US Centers for Disease Control and Prevention, the World Federation for Mental Health, the International Society for Prevention of Child Abuse and Neglect and the World Health Organization, among others. A full list of team members, and brief biographies, is included in Additional file 1, and can be viewed from the Network's website at www.PreVAiLResearch.ca.

To reach its broader goals, PreVAiL had as an initial objective to identify priority research topics and activities based on known gaps in the literature. We conducted a formal priority-setting process, using a modified Delphi approach [16,17], to solicit input from all Network members to identify key research gaps and priorities. The main result of the process, presented in this paper, is a consensus-based list of research priorities that incorporates both the scientific perspective provided by PreVAiL researchers, and the "real world" perspective afforded by PreVAiL knowledge-user partners. Our primary research question, therefore, is "what are the known research gaps in the areas of prevention of child maltreatment and intimate partner violence, and response to these problems, and how should research be prioritized, using a resilience-oriented approach?"

\section{Methods}

\section{Delphi Consensus Development Method}

The Delphi method has been used extensively by health researchers to build consensus on topics such as indicators for monitoring migration and perinatal health [16] and mental health first aid guidelines [18]. It has also proved useful in establishing health and mental health research priorities $[19,20]$. Its primary purpose is to reach consensus on a problem, and it does this through a series of questionnaires administered to an expert panel. The first questionnaire typically presents the problem and collects ideas from participants, which are summarized and used to create a second questionnaire, giving participants a chance to re-evaluate their responses in light of those of others, then rank the items. During this iterative process, the range of answers decreases and the group converges toward a distillation of priorities [17]. We followed this general process, and augmented it with group discussions, organized thematically across our main content areas (CM, IPV, and resilience).

\section{Participants}

All of the original 65 PreVAiL team members (41 researchers, 3 research trainees and 21 knowledge-user partners) were eligible to voluntarily participate in any of the rounds; trainees added as part of PreVAiL's capacity development strategy may also have participated in latter stages of the process. The study received ethical approval from the Western University Health Sciences Research Ethics Board (\#17146E).

\section{Procedures}

Given the international scope of participants, data collection was conducted using password protected online survey software, and teleconferencing, supplemented by emailed forms or comments. The modified Delphi process occurred in three main steps (Figure 1).

\section{Preliminary Phase: Review and Identification of Existing Research Gaps and Priorities}

There were three initial sources of input for the process:

1) PreVAiL had prepared three research briefs (see under “Things We've Done” at www.prevailresearch. ca) in CM, IPV, and RES that summarized the current English-language evidence related to these topics, and identified knowledge gaps. Additional file 2 summarizes these research gaps, by content area.

2) During grant preparation and at early PreVAiL meetings, members identified additional research gaps and priorities, which were extracted from minutes and summaries.

3) At meetings arranged by PreVAiL partners, stakeholders identified their research priorities. For example, at a January 2010 meeting between PreVAiL leads and Government of Canada representatives from the national Family Violence Initiative (FVI) (http://www.phac-aspc.gc.ca/ncfvcnivf/initiative-eng.php), a list of potential research priorities and questions was developed by FVI representatives for potential collaboration with PreVAiL researchers. All identified gaps and priorities from the above were extracted and collated, resulting in 20 starting priorities in the following categories: 1) RES; 2) CM; 3) IPV; 4) issues 
that cross-cut (CC) content areas; and 5) research methods (RM) in these content areas. Survey Rounds 1 and 2: Initial Rating and Ranking of Research Gaps/Priorities.

Round 1 was initiated in Summer 2010 with PreVAiL members receiving an emailed link to the first survey. Participants rated the overall importance of the initial 20 priorities, in the five categories, and could suggest new potential priorities for inclusion in the subsequent round. When rating each priority, participants were asked to think ahead 3 to 5 years and to consider the feasibility and applicability of the research topic: researchers were expected to provide a scientific perspective by reflecting on the feasibility of conducting the research, while knowledge-user partners could provide a 'real world' lens by thinking about the feasibility of applying the research within their context of practice or policy. Participants rated each priority on a 7 -point scale $(1=$ extremely important, $4=$ neutral, and $7=$ not at all important); during scoring, responses were reversed so that higher values indicated greater importance.

The importance ratings from Round 1 as well as comments and suggestions for additional priorities were used to prepare the Round 2 questionnaire, which was administered in Fall 2010. In general, members' comments suggested that more specificity should be used in presenting certain priorities, resulting in further consolidation of "cross-cutting" priorities. Consequently, priorities that had been previously all-inclusive (e.g., in terms of type of violence, setting, etc.) were designated to their respective categories, which in some cases led to an increase in the total number of priorities.

Some members commented that they did not consider themselves qualified to rank priorities in certain categories, therefore "opt out" response options were inserted to allow categories to be skipped (this never exceeded 7 respondents). In Round 2, priorities within each category were presented in the order that they were rated in Round 1, with the most important priorities presented first. New priorities suggested in Round 1 were included at the end of each category along with the explanation for its recommended inclusion (using, anonymously, the words provided by the member making the suggestion). Each priority was listed with a drop-down box beside it so that it could be ranked against the other priorities within that particular category. For example, 11 priorities were listed under the IPV category. Participants ranked each priority, with "1" being the highest and "11" being the lowest ranked. In total, 27 existing priorities and 12 new priorities were ranked in Round 2. To determine rank orders in Round 2, we ran the frequencies for all of the rankings and used the mode to order the final rankings. Ties were indicated after Round 2, but resolved during the Discussion round so that a clear ranked list was produced. All written comments from Round 1 and Round 2 were also summarized and brought forward to the discussion round.

\section{Discussion Round 3: Finalization of Research Gaps/Priorities}

Round 3 consisted of three teleconferences held in April and May, 2011, one for each of RES, CM and IPV, with 
discussion of CC and RM in each. Members were invited by email to sign up for any or all of the discussions; 15 , 14, and 11 participated, with minimal overlap between these groups ( 4 were in all 3,5 in 2 , and 18 in 1). The discussions were used to finalize the priorities in each area, including decisions regarding lower-ranked ones, and how to begin operationalizing top priorities.

\section{Development of Feasibility Themes}

During each round, and especially in Round 3, members were asked to comment on the feasibility of the selected priorities, with researchers asked to focus on issues of conducting the research, and partners on applying/ implementing it in practice and policy settings. These comments were collated according to type (researchversus implementation-specific) and an emerging list of themes developed.

\section{Results}

\section{Survey Rounds 1 and 2}

In total, 44 responses were received in Round 1 and 47 were received in Round 2. The resulting sample (Table 1) comprised a group of national and international researchers and knowledge-user partners, about twothirds of whom were researchers from Canada working at an academic institution, reflecting the initial team composition. The results from Rounds 1 and 2 are presented in Table 2. In each of RES, CM, and IPV, the top-ranked priority was to examine key elements of promising or successful programmes in the area to

Table 1 Participant Characteristics

\begin{tabular}{lcc}
\hline & Round 1\% & Round 2\% \\
\cline { 2 - 3 } & $\mathbf{( N = 4 4 )}$ & \\
\hline Primary Affiliation & $77.3(34)$ & $66.0(31)$ \\
\hline 1. Researcher & $20.5(9)$ & $21.3(10)$ \\
2. Partner & $2.3(1)$ & $12.8(6)$ \\
3. Both & & \\
\hline Work Setting & $70.5(31)$ & $66.0(31)$ \\
\hline 1. Academic Institution & $11.4(5)$ & $14.9(7)$ \\
2. Govt. dept/agency & $2.3(1)$ & $4.3(2)$ \\
3. Non-govt. organization & $11.4(5)$ & $8.5(4)$ \\
4. Research Institute & 0 & $6.4(3)$ \\
5. Other & & \\
\hline Geographic Location & $68.2(30)$ & $14.9(7)$ \\
\hline 1. Canada & $13.6(6)$ & $14.9(7)$ \\
2. United States & $11.4(5)$ & $2.1(1)$ \\
3. Europe & 0 & $4.3(2)$ \\
4. Asia & $6.8(3)$ & $2.1(1)$ \\
5. Australia & 0 & \\
6. Other & &
\end{tabular}

build intervention pilot work $(34.1 \%, 28.9 \%$, and $30 \%$ ranked it first in each of the categories respectively). In the CC category, integrating violence questions into national and international surveys was ranked first, with $40.9 \%$ of participants giving it top priority. In the RM category, the top priority (ranked by $65.1 \%$ ) was to investigate methods for collecting and collating datasets to link data and to conduct pooled, meta- and sub-group analyses to identify promising interventions for particular groups of women, men and children.

\section{Round 3}

In this round, priorities were refined (i.e., reworded, combined, dropped, or reordered) as agreed upon by participants. The final list of priorities can be seen in Table 3. The RES priorities, which included examining the elements underpinning promising or successful programmes in resilience, determining the critical requirements for evidence-based resilience interventions and developing and evaluating interventions to promote resilience in those exposed to CM and/or IPV, did not undergo significant changes. However, the group acknowledged that these are related and could be combined, re-specified, or, particularly with respect to priorities 1 and 2, re-ordered. Participants also noted that it may be necessary to complete some or all of the first two priorities before beginning the third. The need to establish a clear definition of resilience was also raised at several points during that teleconference.

In the CM category, the two priorities involving developing and evaluating new interventions were combined into one broader priority encompassing interventions for primary prevention, recurrence, and associated impairment of CM. In addition, in order to be able to focus efforts on a manageable set of priorities, the 7 bottomranked priorities were dropped from the final list. However, several were discussed at length. For example, the priority focused on CM in Canadian Aboriginal communities was given particular attention. Similarly, due to the large immigrant population in Canada, participants also discussed special considerations related to studying $\mathrm{CM}$ in other racial/ethnic/cultural sub-groups. A footnote regarding studying sub-groups was added to the final priority list, and it was noted that priorities would need to be tailored according to the needs of specific countries, settings, etc.

The IPV priorities underwent more extensive revision. The number of priorities was reduced from 11 to 5 . The bottom-ranked priority from Round 2 ('Controversial issues in IPV') was deemed too difficult to operationalize as a research topic and not specific enough and was therefore dropped. Others were combined because they overlapped in either focus or approach. Finally, participants noted a critical gap in the literature regarding the 


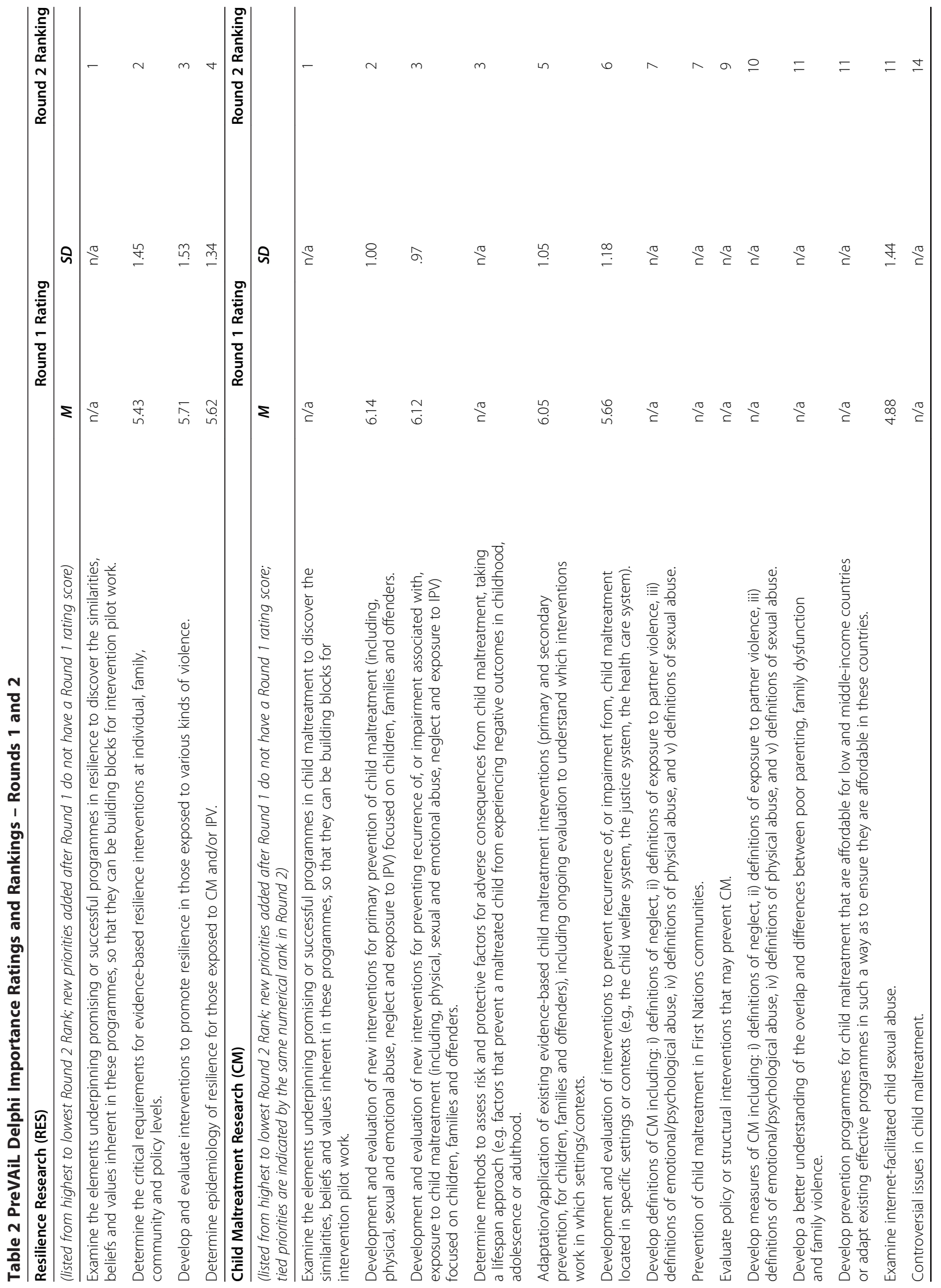




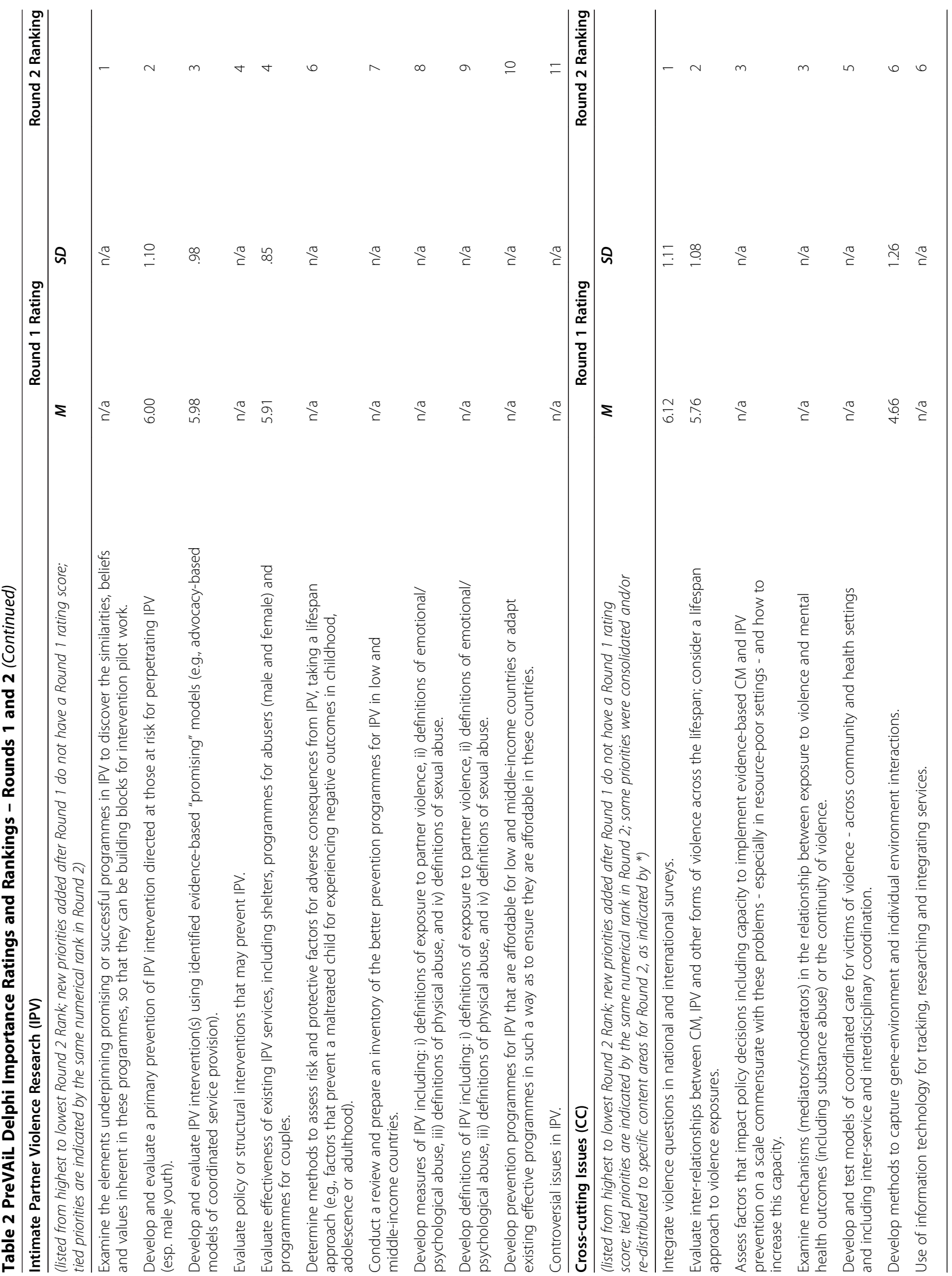




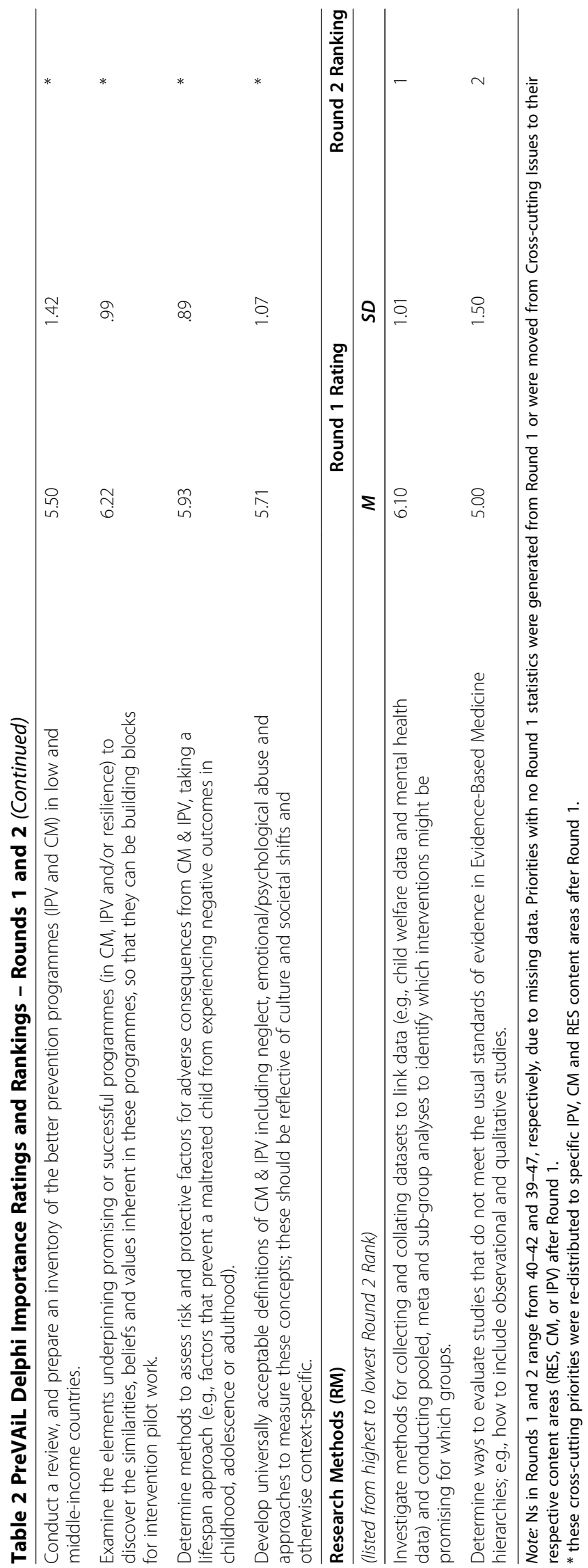




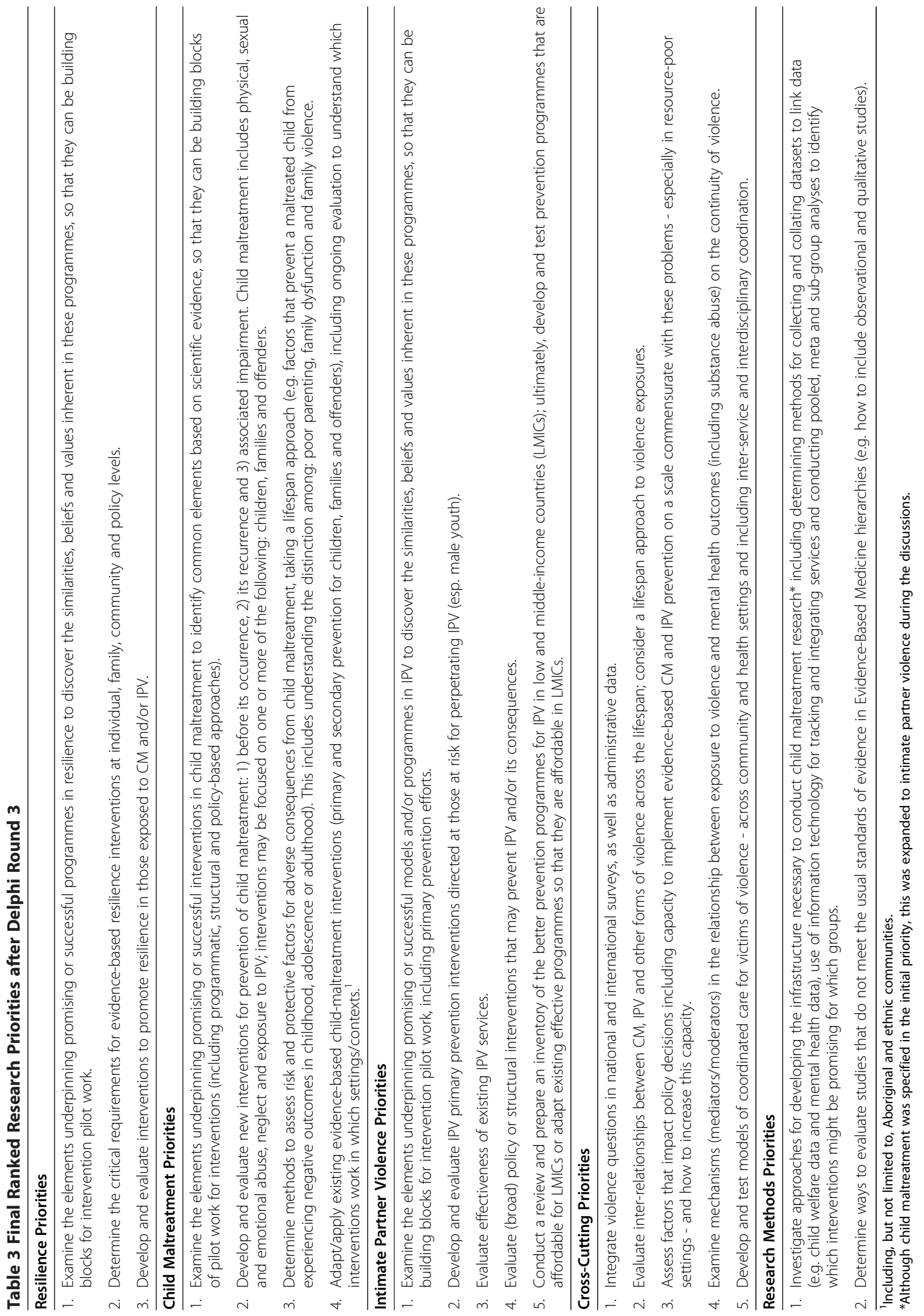




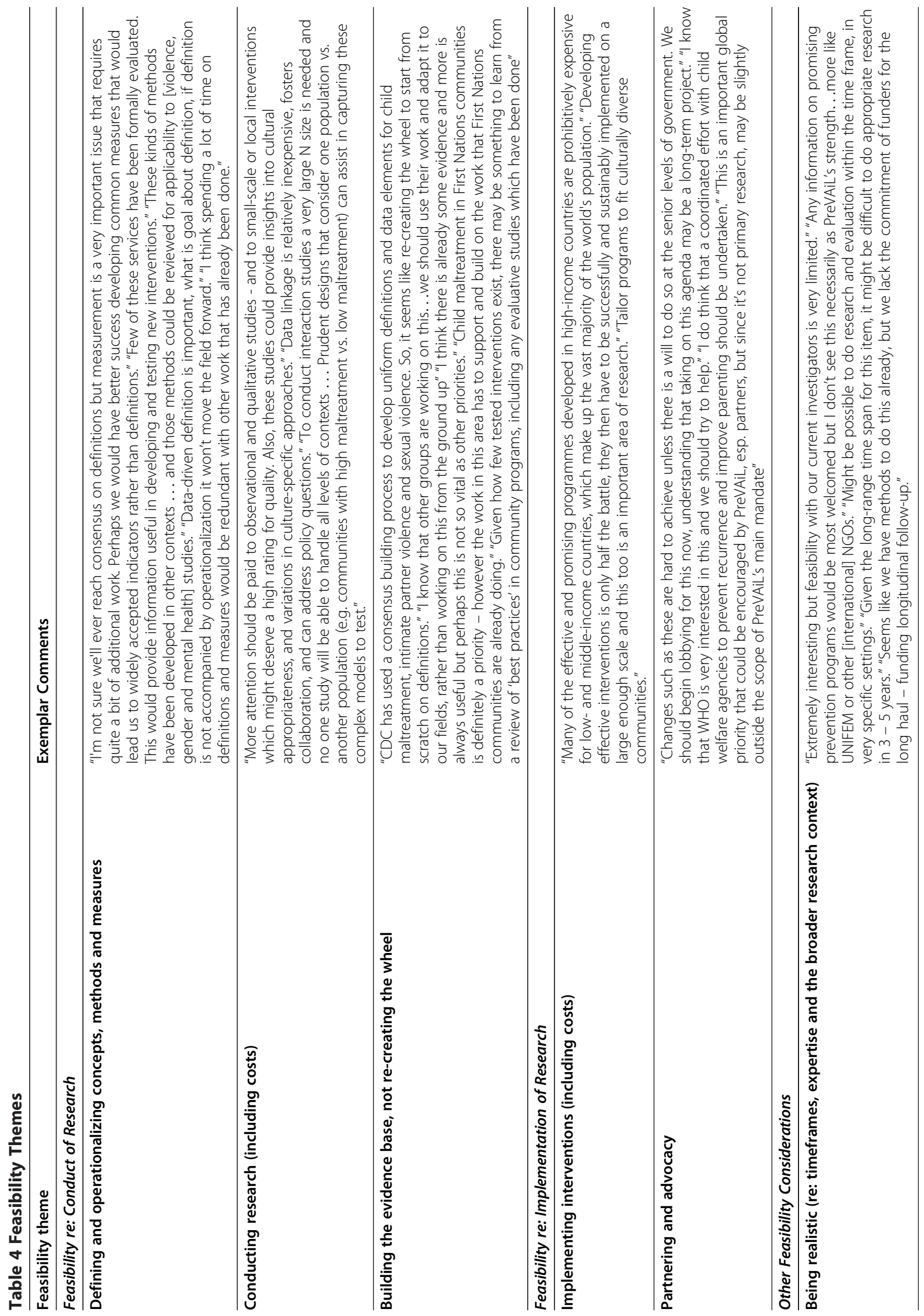


effectiveness of IPV services and so the priority 'Evaluate effectiveness of existing IPV services' was promoted relative to its position in Round 2.

The CC priorities were reduced from 7 to 5 and the concept of better integration of information technology in surveillance was integrated into one of the RM priorities, which otherwise did not change.

\section{Feasibility Themes}

Table 4 presents the themes, grouped according to "research" or "implementation" feasibility, and provides exemplar comments from participants, which addressed issues related to conceptualizing and operationalizing research questions, methods and measures, thinking about how new evidence would build on, rather than replicate, existing efforts, how to consider, in advance, what would need to be put in place to think about implementing research evidence, and being realistic about constraints, including expertise, timing, and the broader research, policy and practice environment.

\section{Discussion}

The results of this consensus development process provide specific guidance to researchers and other stakeholders regarding priority knowledge gaps in the areas of child maltreatment, intimate partner violence and resilience. In all three areas, the top-ranked priority was to examine key elements of promising or successful programmes to build intervention pilot work. This emphasis on intervention development and testing in part reflects the PreVAiL mandate, but is based on the recognized gap in knowledge regarding proven-effective interventions in both CM [8] and IPV [9,10], and the lack of even preliminary intervention work in resilience specific to IPV and CM [14,15]. While promising interventions exist in some areas, these are often based on studies in specific groups and in better-resourced settings. Developing pilot work to take elements from promising existing programmes and services and adapt and test them in new contexts was viewed as an evidence-based, resourceeffective and feasible approach to moving these fields forward. Similarly, in the IPV area, evaluating, using rigourous methods, existing services was a top-three priority.

There was a relatively wide range in the number of priorities identified, in large part reflecting the areas' various stages of development with respect to research. For example, resilience research in the context of violence exposures is in its beginning stages [14,15] and was deemed to require basic definitional and epidemiological work before moving to other kinds of research this was a primary reason for keeping it as a separate thematic area, rather than trying to integrate it as a cross-cutting theme highly relevant to both $\mathrm{CM}$ and
IPV. At a subsequent face-to-face meeting, the resilience theme group discussed at length the conceptual, definitional and methodological challenges in resilience research. They agreed that they viewed resilience as a dynamic life course process that was influenced by interactive individual, biological, social and environmental factors which may assist in the development, maintenance or regaining of mental health despite adversity. The group relied on a broad conceptualization of "mental health", such as that endorsed by the World Health Organization, that went beyond psychopathology to include wellbeing. This led the resilience group to develop and approve the following definition, which will now be used by PreVAiL in its future work:

Resilience is a dynamic process in which psychological, social, environmental and biological factors interact to enable an individual at any stage of life to develop, maintain, or regain their mental health despite exposure to adversity.

While more research is available in CM and IPV, proven-effective interventions exist for only a fraction of possible settings and populations.

The highest degree of consensus was in the methodological category, where $65 \%$ agreed that investigating methods for collecting and collating datasets and conducting pooled, meta and sub-group analyses was the top priority (with appropriate caveats regarding inclusion of only higher-quality studies in these aggregated analyses, and the relative lack of such studies in some areas), along with better technological tools for tracking, surveillance and data linkage. For example, the existence of many high-quality national and international datasets is a potentially rich and efficient starting place to use evolving data linkage techniques [21] to answer questions regarding interrelationships among types of violence, risk and resilience factors, and mental health and addictions, using gender and sex-based analysis methods. Related to this, there was strong support for the integration of violence-specific questions in existing and new national and international surveys, as well as in administrative data sets. Though challenging, moving towards common definitions and questions across content areas would allow meaningful cross-sectional and longitudinal comparisons. There was strong consensus regarding the need to develop innovative ways to think about "evidence" broadly, and to employ rigorous designs of various kinds - quantitative, qualitative and mixed - to problems in these fields, finding appropriate ways to integrate different kinds of data in answering priority questions.

Across priorities, however, there was a recognition that both team-specific and external constraints must be taken into account when considering the feasibility of planning, conducting and implementing research, and this was true 
both from the researcher perspective, as well as from the policy and practice decision-maker partners.

The Delphi method was useful for our purposes for several reasons. First, it is a technique designed specifically to generate consensus from a panel of knowledgeable people. Second, it is a relatively quick and efficient technique, which utilized various communication tools to gather data from our globally-dispersed Network. Potential limitations of the Delphi approach have been noted [22], and Sackman [23], points out that the reliability of measurement and validity of findings using this approach are unknown. Nevertheless, recent critiques [24] have concluded that Delphi is a valuable research method when care is taken with its use; our identification of initial priorities using syntheses of best-available evidence, and known evidence gaps, lends credibility to our process. More quantitative approaches to assessing research priorities are emerging [25,26], which include scoring priorities along specific dimensions, such as significance, answerability, applicability, equity and ethics [27], however, for the purposes of developing priorities within a relatively well-defined scope and among an established research group, the Delphi method yielded results that are specific and relevant, with consideration given to the kinds of dimensions listed above. In addition, beginning the process by building in part on pre-identified research gaps from the PreVAiL Research Briefs (Additional file 2), meant that evidence and systematic reviews based on English-language literature were privileged. However, the priorities we identified through this process complement the broader set of high-profile priorities and "grand challenges" highlighted for global mental health $[26,28,29]$. A potential follow-up to this process would include soliciting feedback from a broader group of identified stakeholders regarding these priorities, both to better align them with those in the broader context, but also to begin building opportunities for ongoing knowledge translation and exchange with those stakeholders.

In terms of lessons learned, the varying types and scope of PreVAiL's expertise meant that some members felt able to provide input on some, but not all, topics, which is a reasonable approach given the scope of PreVAiL's mandate. That said, a group comprised of more tightly-focused expertise in one of these content areas might provide a different set or ordering of priorities. In fact, comments related to feasibility pointed out that PreVAiL's mandate and timeline are potentially limited, and thus, while broadly applicable, some priorities would have to be taken on by others. As one member said:

Addressing violence in low and middle-income countries (LMICs) is an important issue and one that deserves more attention. Whether PreVAiL should take this on or not depends on whether we have investigators willing to build on the inventory to advance intervention work in these countries. If there is no champion within the group, it may not be the best use of PreVAiL time and resources.

Therefore, based in part on discussions that arose during our Delphi process, as well as PreVAiL's membership in the World Health Organization (WHO) Violence Prevention Alliance (VPA), PreVAiL has taken the lead for VPA in conducting a research priority-setting process, using a similar modified Delphi approach, on the topic of interpersonal violence prevention. The goal is to determine a violence prevention research agenda in LMICs, on behalf of WHO VPA, for the next five years. One of the limitations of the PreVAiL process - reliance on a majority of respondents from high-income countries - will be addressed with the VPA survey, which emphasizes participation of representatives from middle and low-income countries. Both have their strengths the PreVAiL-based survey provided an in-depth focus on CM and IPV, while the VPA survey will address additional types of interpersonal violence, including youth violence and elder abuse, but will focus in less depth on the specific types of violence.

An additional challenge when beginning to address these priorities will be how to explicitly build-in a gender and sex-based analysis (GSBA) approach. In cases where the initial priorities suggest evidence synthesis or extraction of best/promising practices from existing interventions, the ability to incorporate GSBA will depend on how those initial data were collected and what is available in various datasets. However, as priorities are implemented that involve developing and testing new interventions, or adding new questions to surveys, etc., explicit incorporation of sex and gender variables and analyses should be prioritized.

\section{Conclusions}

The evidence-based priorities articulated in this paper, which benefit from both a researcher perspective, as well as input from a range of national and international-level policy and practice stakeholders in the area of family violence, can be used as a guide, along with other recent calls for research in mental health $[26,28,29]$, for planning research in prevention of child maltreatment and intimate partner violence, taking a gender and resilience-based perspective.

\section{Additional files}

Additional file 1: Members of the PreVAiL Research Network (see also http://www.prevailresearch.ca/).

Additional file 2: Research Gaps Identified in PreVAiL Research Summaries (see also http://www.prevailresearch.ca/ - "Things We've Done"). 


\section{Competing interests}

The authors have no competing interests to declare.

\section{Authors' contributions}

CNW oversaw data collection, prepared the outline and drafted all sections. $\mathrm{JH}$ and JM assisted with data collection and analysis, and contributed to specific sections of the results. CNW, DES and HLM obtained funding for the PreVAiL Network, and with $\mathrm{JC}$ and $\mathrm{HH}$, are co-principal investigators. All authors participated in staging of Delphi activities, reviewed all sections of the manuscript and provided input on interpretation of results. All authors read and approved the final manuscript.

\section{Acknowledgements}

This research was supported by funds from the Canadian Institutes of Health Research (CIHR) Institute of Gender and Health and Institute of Neurosciences Mental Health and Addictions (INMHA) (grant \#: RDG99326). Nadine Wathen was supported by a CIHR New Investigator Award in Women's Health. Jen MacGregor was supported by a PreVAiL Post-doctoral Fellowship. Harriet MacMillan was supported by the David R. (Dan) Offord Chair in Child Studies. We thank PreVAiL members Gene Feder and Anita Gagnon for methodological advice, and the PreVAiL Advisory Committee for feedback.

\section{Author details}

${ }^{1}$ Faculty of Information \& Media Studies, Western University, London, ON, Canada. ${ }^{2}$ Departments of Emergency Medicine and Community Medicine, West Virginia University, Morgantown, WV, USA. ${ }^{3}$ Department of Psychiatry, University of Melbourne, Melbourne, Australia. ${ }^{4}$ Department of Psychiatry, University of Toronto, Toronto, ON, Canada. ${ }^{5}$ Departments of Psychiatry \& Behavioural Neurosciences, and of Pediatrics, McMaster University, Hamilton, ON, Canada. ${ }^{6}$ McMaster University, Hamilton ON, University of Toronto, Toronto ON, and Western University, London, ON, Canada.

Received: 25 April 2012 Accepted: 4 August 2012

Published: 21 August 2012

\section{References}

1. American Medical Association, Council on Scientific Affairs: Violence against women: relevance for medical practitioners. JAMA 1992, 267(23):3184-3189.

2. Krug EG, Dahlberg LL, Mercy JA, Zwi AB, Lozano R: World report on violence and health. Geneva, Switzerland: World Health Organization; 2002.

3. Rees S, Silove D, Chey T, Ivancic L, Steel Z, Creamer M, et al: Lifetime prevalence of gender-based violence in women and the relationship with mental disorders and psychosocial function. JAMA 2011, 306(5):513-521.

4. Howard LM, Trevillion K, Agnew-Davies R: Domestic violence and mental health. Int Rev Psychiatry 2010, 22(5):525-534.

5. Jordan CE, Campbell R, Follingstad D: Violence and women's mental health: the impact of physical, sexual, and psychological aggression. Annu Rev Clin Psychol 2010, 6:607-628.

6. Gilbert R, Widom CS, Browne K, Fergusson D, Webb E, Janson S: Burden and consequences of child maltreatment in high-income countries. Lancet 2009, 373(9657):68-81.

7. Edwards VJ, Holden GW, Felitti VJ, Anda RF: Relationship between multiple forms of childhood maltreatment and adult mental health in community respondents: results from the adverse childhood experiences study. Am J Psychiatry 2003, 160(8):1453-1460.

8. MacMillan HL, Wathen CN, Barlow J, Fergusson DM, Leventhal JM, Taussig $\mathrm{HN}$ : Interventions to prevent child maltreatment and associated impairment. Lancet 2009, 373(9659):250-266.

9. Feder G, Ramsay J, Dunne D, Rose M, Arsene C, Norman R, et al: How far does screening women for domestic (partner) violence in different health-care settings meet criteria for a screening programme? Systematic reviews of nine UK National Screening Committee criteria. Health Technol Assess 2009, 13(16):iii-iv. xi-xiii, 1-113, 137-347.

10. Sadowski L: Intimate partner violence towards women. Clin Evid 2009, 2(1013):1-15.

11. Wathen $\mathrm{CN}$, MacMillan $\mathrm{HL}$ : Interventions for violence against women: scientific review. JAMA 2003, 289(5):589-600.
12. Nelson HD, Nygren $P$, Mclnerney $Y$, Klein J, and the U.S. Preventive Services Task Force: Screening women and elderly adults for family and intimate partner violence: a review of the evidence for the U.S. Preventive Services Task Force. Ann Intern Med 2004, 140(5):387-396.

13. Ramsay J, Carter Y, Davidson L, Dunne D, Eldridge S, Feder G, et al: Advocacy interventions to reduce or eliminate violence and promote the physical and psychosocial well-being of women who experience intimate partner abuse. Cochrane Database Syst Rev 2009, 3. CD005043.

14. Herrman H, Stewart DE, Diaz-Granados N, Berger EL, Jackson B, Yuen T: What is resilience? Can J Psychiatry 2011, 56(5):258-65.

15. Kolar K: Resilience: Revisiting the concept and its utility for social research. Int J Ment Health Addict 2011, 9(4):421-433.

16. Gagnon AJ, Zimbeck M, Zeitlin J: Migration and perinatal health surveillance: an international Delphi survey. Eur J Obstet Gynecol Reprod Biol 2010, 149(1):37-43.

17. Adler M, Ziglio E (Eds): Gazing into the oracle: The Delphi method and its application to social policy and public health. London: Jessica Kingsley Publishers; 1996

18. Kelly CM, Jorm AF, Kitchener BA: Development of mental health first aid guidelines on how a member of the public can support a person affected by a traumatic event: a Delphi study. BMC Psychiatry 2010, 10:49.

19. Byrne $S$, Wake M, Blumberg D, Dibley M: Identifying priority areas for longitudinal research in childhood obesity: Delphi technique survey. Int $J$ Pediatr Obes 2008, 3(2):120-2.

20. Owens C, Ley A, Aitken P: Do different stakeholder groups share mental health research priorities? A four-arm Delphi study. Health Expect 2008, 11(4):418-31.

21. Clark DE: Practical introduction to record linkage for injury research. Inj Prev 2004, 10:186-191.

22. Powell C: The Delphi technique: myths and realities. J Adv Nurs 2003, 41(4):376-82.

23. Sackman H: Delphi critique. Lexington Books: Lexington; 1975.

24. Vernon W: The Delphi technique: a review. Int J Ther Rehab 2009, 16(2):69-76.

25. Rudan I, Gibson JL, Ameratunga S, El Arifeen S, Bhutta ZA, Black M, et al: Setting priorities in global child health research investments: Guidelines for implementation of the CHNRI Method. Croatian Med J 2008, 49:720-33.

26. Tomlinson M, Rudan I, Saxena S, Swartz L, Tsai AC, et al: Setting priorities for global mental health research. Bull World Health Organ 2009, 87:438-446.

27. Tol WA, Patel V, Tomlinson M, Baingana F, Galappatti A, Panter-Brick C, et al: Research priorities for mental health and psychosocial support in humanitarian settings. PLoS Med 2011, 8(9):e1001096.

28. Lancet Global Mental Health Group, Chisholm D, Flisher AJ, Lund C, Patel V, Saxena S, Thornicroft G, Tomlinson M: Scale up services for mental disorders: a call for action. Lancet 2007, 370(9594):1241-52.

29. Collins PY, Patel V, Joestl SS, March D, Insel TR, Daar AS, Scientific and Advisory Board and the Executive Committee of the Grand Challenges on Global Mental Health: Grand challenges in global mental health. Nature 2011, 475(7354):27-30.

doi:10.1186/1471-2458-12-684

Cite this article as: Wathen et al:: Priorities for research in child maltreatment, intimate partner violence and resilience to violence exposures: results of an international Delphi consensus development process. BMC Public Health 2012 12:684. 